\title{
ESTUDO DA DEGRADAÇÃO DE RESÍDUOS DO CULTIVO DE MILHO PARA A PRODUÇÃO DE ETANOL DE SEGUNDA GERAÇÃO
}

\author{
R. A. P. SILVA ${ }^{1}$, C. A. REZENDE ${ }^{2}$ \\ ${ }^{1}$ Universidade Estadual de Campinas, Faculdade de Engenharia Química \\ ${ }^{2}$ Universidade Estadual de Campinas, Instituto de Química \\ E-mail para contato: r147846@dac.unicamp.br
}

\begin{abstract}
RESUMO - Amostras de resíduos da colheita de milho, como palhas, caules e folhas, foram pré-tratadas em um processo de duas etapas, sendo a primeira com soluções ácidas de $\mathrm{H}_{2} \mathrm{SO}_{4} 1$ e $3 \%$ (v/v) e a segunda com soluções alcalinas de $\mathrm{NaOH} 0,5$ e $5 \%(\mathrm{~m} / \mathrm{v})$, utilizando uma autoclave a $120^{\circ} \mathrm{C}$ e 1,05 bar por $40 \mathrm{~min}$. Também foi realizado um planejamento fatorial para otimizar a retirada de lignina da biomassa, no qual os seguintes fatores foram variados: temperatura (100 e 120 ${ }^{\circ} \mathrm{C}$ ), tempo (40 e $60 \mathrm{~min}$ ) e concentração de $\mathrm{NaOH}(0,5$ e $1 \%)$. Após os prétratamentos, as amostram tiveram seus teores de açúcares, lignina e cinzas quantificados por cromatografia líquida de alta eficiência (HPLC) e sua morfologia analisada por microscopia eletrônica de varredura.
\end{abstract}

\section{INTRODUÇÃO}

Estima-se que cerca de 74 milhões de toneladas de resíduo seco de biomassas são desperdiçadas no mundo por ano e estas teriam o potencial de produzir 49 milhões de litros de bioetanol (Kim, 2003) e outros biocombustíveis (Dias, 2013). Além da questão energética, a biomassa é rica em compostos de carbono, como glicose, frutose, xilose e outros açúcares, que poderiam ser usados na obtenção de produtos químicos de alto valor agregado. Entre esses, destacam-se furfural, hidróximetilfurfural, etanol, butanol, etc. (Stark, 2011; Rinaldi, 2009).

O milho pertence à família Gramineae/Poaceae de nome científico Zea mays L. A planta é basicamente formada pelo caule, as folhas e as espigas. É uma cultura de importância significativa no Brasil e que resulta em uma quantidade grande de biomassa vegetal por tonelada de grãos produzidos. De uma forma geral, a biomassa lignocelulósica é constituída por paredes celulares secas de plantas, cujos principais componentes são celulose, hemicelulose e lignina (Azadi, 2012).

O aproveitamento dos diferentes componentes da parede celular na produção de etanol de segunda geração depende de pré-tratamentos capazes de separá-los e tornar a celulose mais acessível à hidrólise enzimática, uma das etapas determinantes do processo. Assim, esse trabalho está centrado na otimização das etapas de pré-tratamento químico aplicado aos resíduos de milho, buscando teores mínimos de lignina restantes na biomassa e enriquecimento de seu percentual de celulose. 


\section{METODOLOGIA E RESULTADOS}

As amostras de espécies híbridas Zea mays L. (sementes DKB 340 BRO, Dekalb) foram doadas por uma fazenda localizada no município de Casa Branca-SP. Os pés de milho foram colhidos 60 dias após o plantio e moídos em um moinho de facas Thomas Scientific. $\mathrm{O}$ tamanho das partículas foi controlado, utilizando-se uma peneira de $2 \mathrm{~mm}$ de diâmetro, segundo as especificações estabelecidas no protocolo do NREL (Sluiter, 2008). Em todos os pré-tratamentos realizados neste trabalho, as amostram eram uma mistura de folhas, caules e palhas da espiga na proporção $5: 4: 1$. Tal proporção foi determinada de acordo com a massa seca obtida de cada componente da planta.

Os pré-tratamentos utilizados basearam-se em uma metodologia já sólida no grupo de pesquisa (Rezende, 2011). O pré-tratamento consiste de duas etapas: uma primeira etapa ácida, utilizando uma solução de ácido sulfúrico $\left(\mathrm{H}_{2} \mathrm{SO}_{4}\right)$, e uma segunda etapa básica, em que se utiliza um tratamento com hidróxido de sódio $(\mathrm{NaOH})$ aquoso (Rezende, 2011). Foi feito também um tratamento hidrotérmico para comparação, mantendo-se as demais condições iguais à do tratamento ácido-base $\left(120^{\circ} \mathrm{C}, 1,05\right.$ bar e $\left.40 \mathrm{~min}\right)$. Em todos os tratamentos, utilizou-se uma autoclave vertical AV-75 Phoenix. As concentrações utilizadas de cada reagente e os respectivos nomes das amostras estão relacionados na Tabela 1.

Tabela 1 - Relação das amostras de palha de milho tratadas em diferentes concentrações de ácido e base por $40 \mathrm{~min}$, a $120^{\circ} \mathrm{C}$ e 1,05 bar.

\begin{tabular}{|c|c|c|c|}
\hline & Nome da amostra & $\mathbf{H}_{2} \mathbf{S O}_{\mathbf{4}}(\mathbf{v} / \mathbf{v})$ & $\mathbf{N a O H}(\mathbf{m} / \mathbf{v})$ \\
\hline $\mathbf{1}$ & In natura & --- & --- \\
\hline $\mathbf{2}$ & Hidrotérmico & $0 \%$ & --- \\
\hline $\mathbf{3}$ & $\mathrm{H}_{2} \mathrm{SO}_{4} 1 \%$ & $1 \%$ & --- \\
\hline $\mathbf{4}$ & $\mathrm{H}_{2} \mathrm{SO}_{4} 1 \%+\mathrm{NaOH} \mathrm{0,5 \%}$ & $1 \%$ & $0,5 \%$ \\
\hline $\mathbf{5}$ & $\mathrm{H}_{2} \mathrm{SO}_{4} 1 \%+\mathrm{NaOH} \mathrm{5 \%}$ & $1 \%$ & $5 \%$ \\
\hline $\mathbf{6}$ & $\mathrm{H}_{2} \mathrm{SO}_{4} 3 \%$ & $3 \%$ & --- \\
\hline $\mathbf{7}$ & $\mathrm{H}_{2} \mathrm{SO}_{4} 3 \%+\mathrm{NaOH} \mathrm{0,5 \%}$ & $3 \%$ & $0,5 \%$ \\
\hline $\mathbf{8}$ & $\mathrm{H}_{2} \mathrm{SO}_{4} 3 \%+\mathrm{NaOH} 5 \%$ & $3 \%$ & $5 \%$ \\
\hline
\end{tabular}

Os resíduos sólidos obtidos após cada tratamento foram hidrolisados para serem quantificados quanto à sua composição química por HPLC e por espectroscopia UV-Vis, segundo um protocolo descrito anteriormente (Sluiter, 2008). A composição química das amostras encontra-se na Tabela 2.

Analisando a composição das amostras, pode-se observar que a quantidade de hemicelulose diminui quando tratamentos ácidos são aplicados. A quantidade média de hemicelulose nas amostras in natura (1) é de cerca de 6,5\% e esse percentual permanece praticamente inalterado com o tratamento hidrotérmico, que utiliza água apenas. Nos tratamentos usando ácido 1 ou 3\% (3 e 6), o teor de hemicelulose cai para cerca de $2 \%$. Em relação ao percentual de celulose presente nessas amostras, a maioria dos tratamentos tende a enriquecer as amostras em celulose, o que se deve à retirada dos demais componentes da amostra. O único tratamento que diminui o percentual de celulose em relação aos $40 \%$ da 
presentes na amostra in natura (1) é o tratamento hidrotérmico. Nos tratamentos com altas concentrações de $\mathrm{NaOH}$ (5 e 8), a amostra final é composta essencialmente por celulose pura (percentuais entre 94 e $99 \%$ ), embora seja importante considerar a perda de massa total nesses processos, que é de $75 \%$. Esses tratamentos mostram-se assim bastante efetivos na obtenção de substratos ricos em celulose para produção de etanol de segunda geração.

Tabela 2 - Composição química das amostras de milho antes e após os pré-tratamentos.

\begin{tabular}{|c|c|c|c|c|c|c|}
\hline \multicolumn{2}{|c|}{ Amostra } & Celulose & Hemicelulose & Lignina & Cinzas & Total \\
\cline { 3 - 7 } & Média & Média & Média & Média & Média \\
\hline $\mathbf{1}$ & In natura A (extrativos) & $41 \pm 1$ & $6,6 \pm 0,3$ & $28,0 \pm 0,5$ & $0,65 \pm 0,02$ & $99 \pm 2$ \\
\hline $\mathbf{3}$ & $\mathbf{H}_{\mathbf{2}}$ & $39,4 \pm 0,9$ & $6,1 \pm 0,7$ & $29,7 \pm 0,3$ & $0,53 \pm 0,01$ & $76 \pm 1$ \\
\hline $\mathbf{4}$ & $\mathbf{H}_{\mathbf{2}} \mathbf{S O}_{\mathbf{4}} \mathbf{1 \%}+\mathbf{S O} \mathbf{1 \%}$ & $54,1 \pm 0,2$ & $2,9 \pm 0,2$ & $34,18 \pm 0,09$ & $1,35 \pm 0,01$ & $92,5 \pm 0,3$ \\
\hline $\mathbf{5}$ & $\mathbf{H}_{\mathbf{2}} \mathbf{S O}_{\mathbf{4}} \mathbf{1 \%}+\mathbf{N a O H ~ 0 , 5 \%}$ & $73 \pm 6$ & $2,5 \pm 0,3$ & $26 \pm 1$ & $0,56 \pm 0,03$ & $102 \pm 5$ \\
\hline $\mathbf{6}$ & $\mathbf{H}_{\mathbf{2}} \mathbf{S O}_{\mathbf{4}} \mathbf{3 \%}$ & $58,13 \pm 0,01$ & $2,42 \pm 0,04$ & $35,5 \pm 0,9$ & $1,49 \pm 0,05$ & $97,5 \pm 0,8$ \\
\hline $\mathbf{7}$ & $\mathbf{H}_{\mathbf{2}} \mathbf{S O}_{\mathbf{4}} \mathbf{3 \%}+\mathbf{N a O H ~ 0 , 5 \%}$ & $79 \pm 12$ & $2,0 \pm 0,2$ & $25,9 \pm 0,6$ & $0,41 \pm 0,01$ & $107 \pm 12$ \\
\hline $\mathbf{8}$ & $\mathbf{H}_{\mathbf{2}} \mathbf{S O}_{\mathbf{4}} \mathbf{3 \%}+\mathbf{N a O H ~ 5 \%}$ & $99 \pm 9$ & $2,0 \pm 0,2$ & $9 \pm 1$ & $0,33 \pm 0,00$ & $110 \pm 8$ \\
\hline
\end{tabular}

*No caso da amostra in natura, o total é obtido somando-se também os 22,5\% de extrativos, retirados com $\mathrm{H}_{2} \mathrm{O}$ e etanol/hexano (1:1) em extrator Sohlet.

O principal função dos tratamentos com $\mathrm{NaOH}$ foi retirar lignina da biomassa, já que esse componente tem a função de conferir rigidez, impermeabilidade e resistência à planta. Assim, a menor quantidade de lignina resulta em maior disponibilidade, acessibilidade e área superficial para as enzimas que atuarão na etapa de hidrólise enzimática para produção de etanol. De acordo com a Tabela 2, pode-se afirmar que a biomassa do milho in natura (1) possui cerca de $30 \%$ de lignina em sua composição. O tratamento hidrotérmico tem pouco efeito sobre esse percentual, mas o teor de lignina cai para cerca de $25 \%$ quando é utilizado, na segunda etapa do pré-tratamento, uma solução alcalina de concentração $0,5 \%$ (4 e 7), independentemente da concentração da solução ácida utilizada na etapa anterior. Já para as soluções de $\mathrm{NaOH}$ 5\% (5 e 8), o valor cai para cerca de 10\% e também independe da concentração do ácido utilizado na primeira etapa. No caso das amostras tratadas apenas com ácido (3 e 6), o percentual de lignina parece aumentar em relação à amostra in natura, mas isso se deve, na verdade, à retirada de outros componentes presentes na biomassa, principalmente hemicelulose. Com essa análise, observa-se que a retirada de lignina do material está intrinsecamente relacionada à concentração da solução alcalina, ou seja, o $\mathrm{NaOH}$ é o responsável pelo aumento da acessibilidade da biomassa por seu efeito de extrair lignina.

Procurando compreender melhor quais dos fatores de processo (temperatura, tempo e concentração de $\mathrm{NaOH}$ ) tem maior influência sobre a concentração mínima final de lignina nas amostras, montou-se um planejamento fatorial $2^{3} \mathrm{em}$ duplicata. Os dados do planejamento, incluindo os níveis em que cada um dos fatores foram variados e a quantidade de lignina total obtida (solúvel e insolúvel) após os tratamentos encontram-se na Tabela 3. O cálculo dos efeitos principais e das interações entre os efeitos encontram-se na Tabela 4. 
Tabela 3 - Dados do planejamento fatorial.

\begin{tabular}{|c|c|c|c|c|c|c|}
\hline \multirow{3}{*}{\multicolumn{4}{|c|}{\begin{tabular}{|l|l|} 
Fatores: & 1: Temperatura, ${ }^{\circ} \mathrm{C}$ \\
& 2: Tempo, min \\
3: Concentração $\mathrm{NaOH}, \%(\mathrm{~m} / \mathrm{v})$
\end{tabular}}} & \multicolumn{2}{|c|}{$100(-)$} & \multirow{2}{*}{$\begin{array}{c}120(+) \\
60(+)\end{array}$} \\
\hline & & & & \multicolumn{2}{|c|}{$40(-)$} & \\
\hline & & & & \multicolumn{2}{|c|}{$0,5(-)$} & $\mathbf{1 , 0}(+)$ \\
\hline Ensaio & 1 & 2 & 3 & Quantidad & ignina $(\%)$ & Média (\%) \\
\hline 1 & - & - & - & 25,21 & 24,74 & $25,0 \pm 0,3$ \\
\hline 2 & + & - & - & 24,94 & 25,34 & $25,1 \pm 0,3$ \\
\hline 3 & - & + & - & 24,13 & 25,49 & $25 \pm 1$ \\
\hline 4 & + & + & - & 31,33 & 32,01 & $31,7 \pm 0,5$ \\
\hline 5 & - & - & + & 15,22 & 14,46 & $14,8 \pm 0,5$ \\
\hline 6 & + & - & + & 17,08 & 16,82 & $17,0 \pm 0,2$ \\
\hline 7 & - & + & + & 16,39 & 16,27 & $16,33 \pm 0,09$ \\
\hline 8 & + & + & + & 17,08 & 17,16 & $17,12 \pm 0,05$ \\
\hline
\end{tabular}

Tabela 4 - Efeitos principais e de interação entre os fatores.

\begin{tabular}{|c|c|c|c|c|c|c|}
\hline $\mathbf{1}$ & $\mathbf{2}$ & $\mathbf{3}$ & $\mathbf{1 2}$ & $\mathbf{1 3}$ & $\mathbf{2 3}$ & $\mathbf{1 2 3}$ \\
\hline $2,5 \pm 0,2$ & $2,0 \pm 0,2$ & $-10,3 \pm 0,2$ & $1,3 \pm 0,2$ & $-1,0 \pm 0,2$ & $-1,2 \pm 0,2$ & $2,0 \pm 0,2$ \\
\hline
\end{tabular}

Analisando os dados, temos que a temperatura (1) e o tempo de residência (2) na autoclave não possuem um efeito principal significativo na retirada de lignina, dentro dos intervalos estudados (temperatura entre 100 e $120^{\circ} \mathrm{C}$ e tempo entre 40 e $60 \mathrm{~min}$ ). No entanto, o efeito principal 3, o qual corresponde à concentração de $\mathrm{NaOH}$, é significativo e se destaca dentre todos os outros efeitos de interação. Isso significa que, quando mudamos do patamar baixo da concentração $(0,5 \mathrm{~m} / \mathrm{v})$ para o mais alto $(1,0 \mathrm{~m} / \mathrm{v})$ temos uma média de diminuição de $10 \%$ na quantidade de lignina da amostra e não há evidências de que esse aumento dependa dos níveis das outras variáveis, na faixa experimental investigada.

O ensaio que revelou a menor quantidade de lignina foi o ensaio 5 , com uma média de $14,8 \%$, o qual foi realizado a $100{ }^{\circ} \mathrm{C}(-)$, por 40 min (-) a uma concentração de $\mathrm{NaOH} 1,0 \%$ $(+)$, enquanto que o ensaio com $120^{\circ} \mathrm{C}(+)$, por $60 \min (+)$ a mesma concentração teve uma média de $17,12 \%$ de lignina na composição. Tais dados confirmam que o tempo de residência na autoclave e a temperatura não possuem um efeito significativo na retirada da lignina. Dessa forma, é possível utilizar tempos relativamente curtos e temperaturas menores nos tratamentos, visando economia de energia.

Para a análise morfológica dos resíduos de milho, estes foram separados em caule, folhas e palha (da espiga) para facilitar a identificação das variações morfológicas em decorrência dos tratamentos. A Figura 1 mostra fibras de palha de milho com sinais brandos de degradação nas amostras tratadas só com $\mathrm{H}_{2} \mathrm{SO}_{4} \quad 1 \%$ (Figura 1B) ou com $\mathrm{H}_{2} \mathrm{SO}_{4} \quad 1 \%+$ $\mathrm{NaOH}$ 0,5\% (Figura 1C), mas uma morfologia bastante degradada pelo tratamento com $\mathrm{H}_{2} \mathrm{SO}_{4} 1 \%+\mathrm{NaOH} 5 \%$ (Figura 1D). Na Figura 1(A), observa-se com clareza as fronteiras das paredes celulares das células que compõem a palha da espiga e também uma fina camada de tecido que protege tais células, além de alguns estômatos espalhados ao longo dele. Já na 
Figura 1(B), nessa amostra pré-tratada com ácido sulfúrico 1\%, observam-se tecidos dobrados e rupturas na parede celular, que caracterizam as consequências morfológicas do tratamento químico aplicado. Na Figura 1(D), as estrutura fibrilar do milho (que é uma gramínea) está solta e as fibras menores encontram-se emaranhadas e retorcidas. A separação do feixe de fibras em amostras de gramíneas foi anteriormente observada nesse grupo de pesquisa (Rezende, 2011).

(A) Palha da espiga - In natura

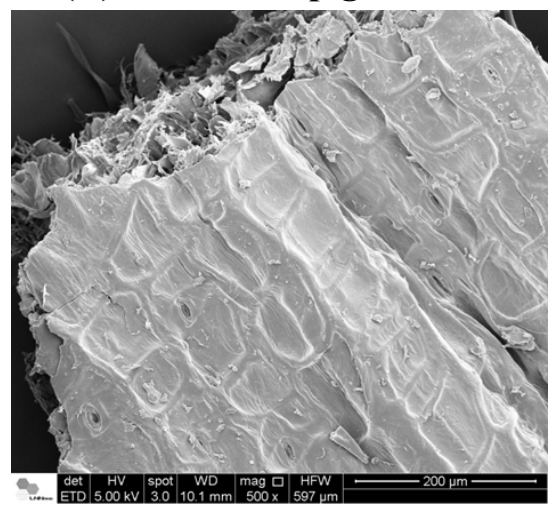

(C) Palha da espiga $\mathrm{H}_{2} \mathrm{SO}_{4} 1 \%+\mathrm{NaOH} 0,5 \%$

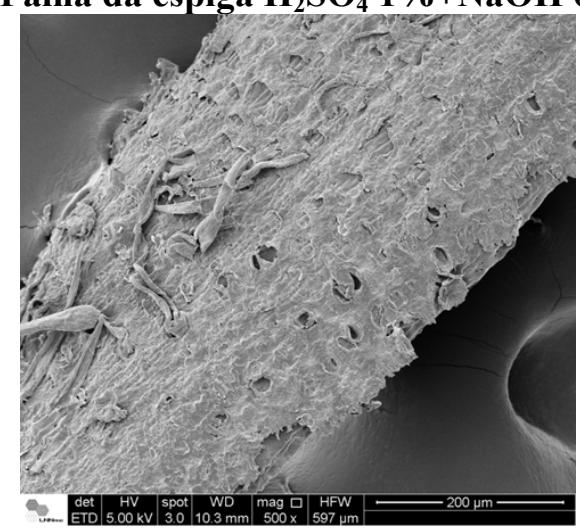

(B) Palha da espiga $\mathrm{H}_{2} \mathrm{SO}_{4} 1 \%$

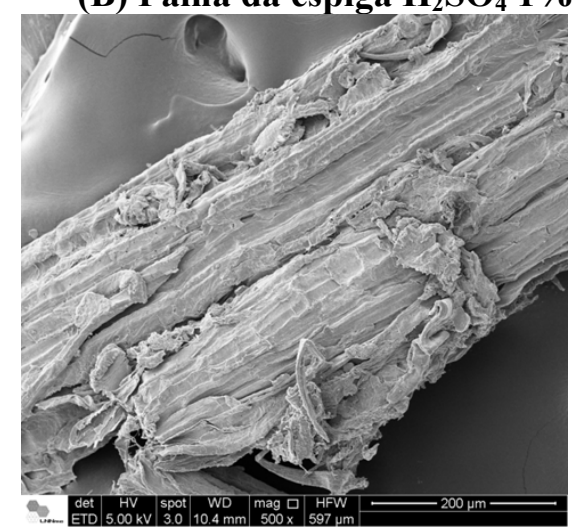

(D) Palha da espiga $\mathrm{H}_{2} \mathrm{SO}_{4} 1 \%+\mathrm{NaOH} 5 \%$

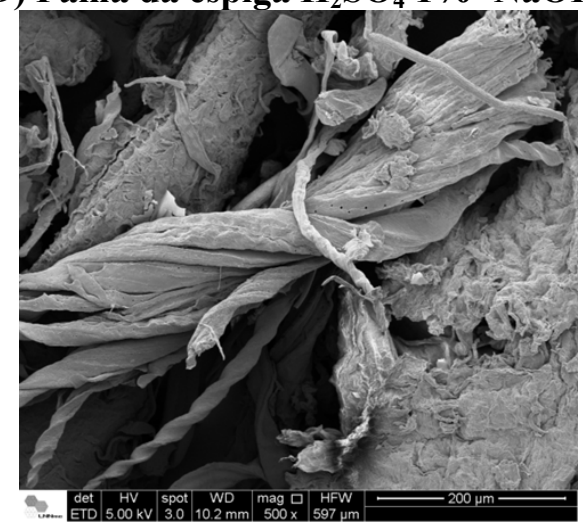

Figura 1. Imagens obtidas por FESEM da palha da espiga do milho: (A) in natura; (B) após tratamento com $\mathrm{H}_{2} \mathrm{SO}_{4} 1 \%$; $\left(\mathrm{C} \mathrm{H}_{2} \mathrm{SO}_{4} 1 \%+\mathrm{NaOH} 0,5 \%\right.$; e (D) $\mathrm{H}_{2} \mathrm{SO}_{4} 1 \%+\mathrm{NaOH} 5 \%$. Barra de escala de $200 \mu \mathrm{m}$.

Esses resultados morfológicos estão totalmente coerentes com os dados de retirada de lignina apresentados na Tabela 2. O tratamento mais brando retira pouca lignina, deixando acessível algumas partes das estruturas fibrilares, por outro lado, o tratamento severo ataca fortemente a lignina e a remove com tanta significância que o material fica sem suporte e sem rigidez, perdendo totalmente a sua forma original. A Figura 2 mostra imagens ampliadas da parede celular da palha de milho em que os contornos das fibrilas individuais de celulose começam a se tornar nítidos. A superfície relativamente compacta da amostra in natura (Figura 2A) começa a apresentar poros maiores após os tratamentos químicos, o que pode ser atribuído à retirada de componentes da parede celular. Essas imagens em ampliações maiores evidenciam as mudanças morfológicas em uma escala bastante ampliada e mostram que a retirada de hemicelulose e de lignina dos interstícios entre as fibrilas e celulose, evidencia o relevo das mesmas. 


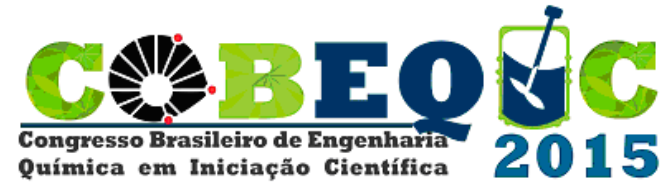

(A) Palha da espiga - In natura

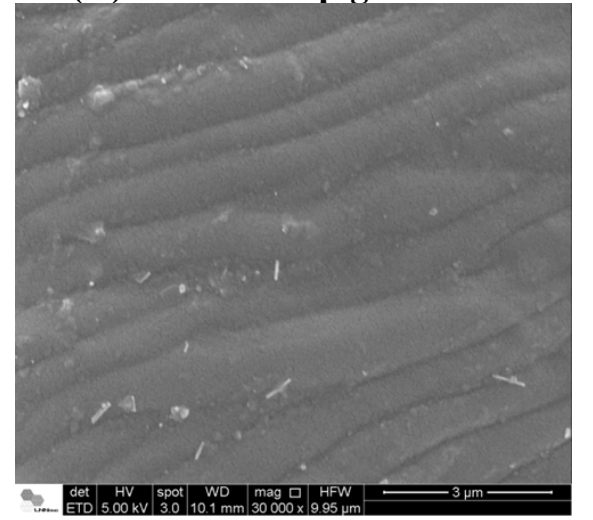

XI Congresso Brasileiro de Engenharia

Química em Iniciação Científica

Unicamp - Campinas - SP

19 a 22 de julho de 2015

Figura 2. Imagens obtidas por FESEM da palha da espiga do milho: (A) in natura; (B) após tratamento com $\mathrm{H}_{2} \mathrm{SO}_{4} 1 \%+\mathrm{NaOH} 5 \%$. Barra de escala de $3 \mu \mathrm{m}$.

\section{CONCLUSÕES}

Os tratamentos químicos aplicados nesse trabalho mostram-se muito efetivos para retirar lignina dos resíduos de biomassa de milho e para a obtenção de substratos enriquecidos em celulose para a obtenção de etanol de segunda geração. O pré-tratamento em duas etapas com $\mathrm{H}_{2} \mathrm{SO}_{4} 3 \%+\mathrm{NaOH} 5 \%$ rendeu as maiores porcentagens de celulose e as menores de lignina, além de apresentar uma morfologia mais aberta e com grande área de contato para hidrólise enzimática. O planejamento fatorial $2^{3}$ mostrou que a concentração de $\mathrm{NaOH}$ é o efeito principal mais relevante para retirada da lignina das amostras, em detrimento do tempo e da temperatura de reação, dentro das faixas utilizadas neste trabalho.

Agradecimento: os autores agradecem ao LME/LNNano/CNPEM pelo uso do microscópio eletrônico de varredura.

\section{REFERÊNCIAS}

AZADI, P.; INDERWILDI, O. R.; FARNOOD, R.; KING, D. A. Liquid fuels, hydrogen and chemicals from lignin: A critical review, Renewable and Reviews, 2012, Sustainable Energy

DIAS, M. O. S.; JUNQUEIRA, T. L.; CUNHA, M. P.; BONOMIA, A.; MACIEL FILHO, R.; Utilization of pentoses from sugarcane biomass: Techno-economics of biogas vs. butanol production", Bioresource Technology, Volume 142, 2013.

KIM, S.; DALE, E. D. Global potential bioethanol production from wasted crops and crop residues, Biomass and Bioenergy, Vol. 26, p. 361-375, 2003.

REZENDE, C. A.; DE LIMA, M. A.; MAZIERO, P.; DE AZEVEDO, E. R.; GARCIA, W.;

POLIKARPOV. I. Chemical and morphological characterization of sugarcane bagasse submitted to a delignification process for enhanced enzymatic digestibility. Biotechnology for Biofuels 4, $54,2011$.

RINALDI, R. et. Al. Acid hydrolysis of cellulose as the entry point into the biorefinery schemes, Chem. Sus. Chem., 2, p. 1096, 2009.

SLUITER, J.; SLUITER, A. Preparation of Samples for Compositional Analysis", Laboratory Analytical Procedure, NREL/TP-510-42620, revised August 2008.

STARK, A. Ionic liquids in the biorefinery: a critical assessment of their potential, Energy Environ. Sci. 4, p. 19, 2011. 